\title{
Editorial
}

\section{Salpingectomía de oportunidad}

\author{
Dr. Omar Nazzal Nazal
}

Ginecólogo Oncólogo Profesor Asociado Facultad Medicina Universidad de Chile Campus Centro. Unidad de Ginecología Oncológica Hospital Clínico San Borja Arriaran. Jefe Técnico Ginecología Integramédica. FACOG. Past President de SOCHOG.

El cáncer de ovario tiene la mas alta mortalidad entre todos los canceres ginecológicos y es la quinta causa de muerte por cáncer en la mujer en el mundo. En Chile según Globocan 2018 se diagnosticaron 837 casos y 549 muertes 1 .

La sobrevida global por esta enfermedad ha presentado mejorías marginales en los últimos 50 años. Los carcinomas epiteliales de alto grado del ovario representan cerca del $90 \%$ del total de casos y concentran sobre el $90 \%$ de mortalidad 2 .

Es sabido el nulo aporte de la ultrasonografía y los marcadores tumorales en el tamizaje de estos tumores en la población de riesgo poblacional3.

En las ultimas décadas se ha generado abundante información relacionada con la carcinogénesis de los tumores epiteliales de alto grado, sabiéndose hoy que la mayoría de los carcinomas serosos de alto grado, endometriodes y de células claras del ovario derivan de la trompa y no directamente del ovario. En mujeres con predisposición genética para cáncer de ovario se han encontrado lesiones intraepiteliales en la trompa que en todo semejan a los carcinomas serosos de alto grado del ovario. Estudios moleculares demuestran que estas lesiones tubáricas expresan una mutación de TP53 tal cual lo hacen los tumores serosos de alto grado. A raíz de esto se ha desarrollado una teoría en relación con que la salpingectomía de oportunidad ofrecería una excelente alternativa para disminuir el riesgo de cáncer de ovario entre aquellas mujeres que se sometan a una cirugía pélvica por una causa benigna $e$ incluso en procedimientos de esterilización ${ }^{4-8}$.

Aun así, se debe tener presentes que otros tumores epiteliales del ovario, así como los originados en las células germinales y en el estroma sin son originarios del ovario. Por ello la contribución está destinada a solo un grupo de ellos, pero sin dudas los más frecuentes y relevantes.

Las investigaciones en este campo han estado enfocadas en dos grupos de mujeres, aquellas con alto riesgo de cáncer hereditario del ovario principalmente mutaciones genes BRCA 1 y $2-\mathrm{y}$ aquellas con riesgo estándar y que son intervenidas por alguna causa benigna de cirugía en la pelvisprincipalmente esterilización tubaria e histerectomía-

La salpingoligadura tiene un conocido rol protector especialmente en aquellos tumores habitualmente relacionados con menstruación retrograda (endometroides y células claras) ${ }^{9}$.

En el clásico estudio de las enfermeras se demostró una reducción del riesgo de un $24 \%$ asociado a su realización ${ }^{10}$. De igual manera cuando se realiza salpingectomía bilateral en adición a otra cirugía pélvica por ejemplo histerectomía, el riesgo de cáncer de ovario se reduce aun más; estudios suecos demostraron disminución del riesgo de hasta un $65 \% 11$.

La salpingectomía de oportunidad aparece entonces como un real aporte en disminuir el riesgo futuro de cáncer de ovario.

De allí, entonces aparece como relevante conocer la seguridad asociada a su realización, la evidencia la señala como un procedimiento seguro que no incrementa los riesgos de complicaciones (necesidad de transfusiones, tasa de infección, fiebre, prolongación excesiva de los tiempos quirúrgicos $\mathrm{ni}$ readmisión) así como tampoco se ha demostrado una alteración en la función ovárica post procedimiento utilizando diferentes métodos para asegurar esto (validaciones de hormona antimulleriana, $\mathrm{FSH}$, recuentos de folículos o incluso respuesta a procedimientos de fertilización asistida) ${ }^{12}$. 
A pesar de estos argumentos no existe aun un completo consenso en la práctica ginecológica con respecto a estos argumentos, tanto así por la natural resistencia al cambio tan enraizada en alguna prácticas médicas como así también por reportes que entregan algunos datos diferentes a los antes planteados, especialmente en lo relacionado a repercusiones endocrinas y síntomas climatéricos en la población afecta al procedimiento ${ }^{13-14}$. Es por dichas circunstancias que no solo es importante entregar estos antecedentes a la comunidad médica, sino sobre todo conocer cuales son sus respuestas ante estas y por ello la relevancia del trabajo de Munera y colaboradores en esta Edición en donde muestran la realidad de Colombia en base a una encuesta realizada al interior de la Sociedad Colombiana de Ginecología y Obstetricia, en lo que respecta a salpingectomía de oportunidad en pacientes con riesgo poblacional, en donde a pesar de recoger solo un $20 \%$ de respuestas del universo buscado, encontraron importantes conclusiones donde un alto porcentaje de los que responden manifiestan que si lo realizan y cuya principal motivación es la reducción del cáncer de ovario y esto basado principalmente en el conocimiento actual, las recomendaciones de organizaciones como American College of Obstetricians and Gynecologists (ACOG) o sugerencia de colegas Ginecólogos Oncólogos, de igual manera las barreras para su no ejecución son las mismas, no aumentar riesgos quirúrgicos intraoperatorios o miedo a alterar la función ovárica.

Como consideraciones finales es importante recalcar que diferentes organizaciones internacionales como el ACOG avalan este tipo de nuevas recomendaciones y sugieren:

- Que la salpingectomía al momento de la histerectomía o la esterilización tubaria parece ser segura y no aumenta el riesgo de complicaciones.

- La función ovárica parece no verse afectada por el procedimiento

- Que como toda practica médica actual, esta debe ser evaluada junto a la mujer y debe incluir un consentimiento informado acorde a la intervención

- La salingectomía postparto o cesárea parece factible y segura

- Planificar la salpingectomía de oportunidad no debe alterar la vía planeada de abordaje quirúrgico ${ }^{15}$.

\section{REFERENCIAS}

1. The Globocan Cancer Observatory, December 2020

2. Matz M, Coleman MP, Sant M, et al the histology of ovarian cancer: worldwide distribution and implications for international survival comparisons (CONCORD -2) Gynecol Oncol 2017;144:403-13

3. The role of the obstetrician-gynecologist in the early detection of epithelial ovarian cancer in women at average risk. Committee Opinion $\mathrm{N}^{\circ}$ 716 American College of Obstetricians and Gynecologists. Obstet Gynecol 2017; 130:146-9

4. Kurman RJ, Shih I. The dualistic model of ovarian carcinogenesis: revisited, revised, and expanded. Am J Pathol 2016; 186:733-47.

5. Erickson BK, Conner MG, Landen CN Jr. The role of the fallopian tube in the origin of ovarian cancer. Am J Obstet Gynecol 2013;209:409-14.

6. Crum CP. Intercepting pelvic cancer in the distal fallopian tube: theories and realities. Mol Oncol 2009; 3:165-70.

7. Kindelberger DW, Lee $\mathrm{Y}$, Miron A, Hirsch MS, Feltmate $\mathrm{C}$, Medeiros $\mathrm{F}$, et al. Intraepithelial carcinoma of the fimbria and pelvic serous carcinoma: evidence for a causal relationship. Am J Surg Pathol 2007; 31:161-9.

8. Kuhn E, Kurman RJ, Vang R, Sehdev AS, Han G, Soslow R, et al. TP53 mutations in serous tubal intraepithelial carcinoma and concurrent pelvic high-grade serous carcinoma evidence supporting the clonal relationship of the two lesions. J Pathol 2012; 226:421-6.

9. Garavaglia E, Sigismondi C, Ferrari S, et al. The origin of endometriosis-associated ovarian cancer from uterine neoplastic lesions. Med Hypotheses 2018; 110:80-2.

10. Rice MS, Hankinson SE, Tworoger SS. Tubal ligation, hys- terectomy, unilateral oophorectomy, and risk of ovarian cancer in the Nurses' Health Studies. Fertil Steril 2014; 102:192-8. e3.

11. Falconer $\mathrm{H}$, Yin L, Gronberg $\mathrm{H}$, Altman D. Ovarian cancer risk after salpingectomy: a nationwide population-based study. J Natl Cancer Inst 2015;107(2): dju410.

12. Hanley GE, McAlpine JN, Pearce CL, Miller D. The per- formance and safety of bilateral salpingectomy for ovarian cancer prevention in the United States. Am J Obstet Gynecol 2017; 216:270. e1-9 
13. Zhen Yuan,Dongyan Cao. Xiaoning Bi, Mei Yu,Jiaxin Yang,Keng SheThe effects of hysterectomy with bilateral salpingectomy on ovarian reserve Int J Gynecol Obstet 2019; 145: 233-238

14. Collins E, Strandell A, Granåsen G, et al. Menopausal symptoms and surgical complications after opportunistic bilateral salpingectomy, a register-based cohort study. Am J Obstet Gynecol 2019; 220:85. e1-10.

15. Opportunistic salpingectomy as a strategy for epithelial ovarian can- cer prevention. ACOG Committee Opinion No. 774. American College of Obstetricians and Gynecologists. Obstet Gynecol 2019;133:e279-84. 